\title{
Application of the Feynman-tree theorem together with BCFW recursion relations
}

\author{
M. Maniatis ${ }^{1, *}$ \\ ${ }^{1}$ Departamento de Ciencias Básicas, UBB, Casilla 447, Chillán, Chile.
}

\begin{abstract}
Recently, it has been shown that on-shell scattering amplitudes can be constructed by the Feynman-tree theorem combined with the BCFW recursion relations. Since the BCFW relations are restricted to tree diagrams, the preceding application of the Feynman-tree theorem is essential. In this way amplitudes can be constructed by on-shell and gauge-invariant tree amplitudes. Here we want to apply this method to the electron-photon vertex correction. We present all the single, double, and triple phase-space tensor integrals explicitly and show that the sum of amplitudes coincides with the result of the conventional calculation of a virtual loop correction.
\end{abstract}

\section{INTRODUCTION}

The Feynman-tree theorem has been introduced by R. P. Feynman decades ago [1, 2]. The idea is to consider besides the usual Feynman propagators $G_{F}(p)$ advanced propagators $G_{A}(p)$,

$$
G_{F}(p)=\frac{i}{p^{2}-m^{2}+i \epsilon}, \quad G_{A}(p)=\frac{i}{p^{2}-m^{2}-i \epsilon \operatorname{sgn}\left(p_{0}\right)} .
$$

With help of the identity $\frac{1}{x \pm i \epsilon}=P . V .\left(\frac{1}{x}\right) \mp i \pi \delta(x)$, where P.V. is the principal value prescription we have

$$
G_{A}(p)=G_{F}(p)-2 \pi \delta^{(+)}\left(p^{2}-m^{2}\right)
$$

with $\delta^{(+)}\left(p^{2}-m^{2}\right)=\theta\left(p_{0}\right) \delta\left(p^{2}-m^{2}\right)$, as usual. Consider a loop with the usual Feynman propagators $G_{F}(p)$ replaced by the advanced propagators $G_{A}(p)$. In the loop momentum integration the poles of the zero component lie therefore all above the real axis. Closing the integration contour in the lower half plane, we get the Feynman-tree theorem:

$$
\begin{aligned}
0 & =\int \frac{d^{4} q}{(2 \pi)^{4}} N(q) \prod_{i} G_{A}^{(i)}\left(q-p_{1}-\ldots-p_{i}\right) \\
& =\int \frac{d^{4} q}{(2 \pi)^{4}} N(q) \prod_{i}\left\{G_{F}^{(i)}\left(q-p_{1}-\ldots-p_{i}\right)-2 \pi \delta^{(+)}\left(\left(q-p_{1}-\ldots-p_{i}\right)^{2}-m^{2}\right)\right\} .
\end{aligned}
$$

Expanding the product on the right-hand side of the last equation, the loop diagram is expressed in terms of cut diagrams, where each cut is given by the corresponding delta distribution. The function $N(q)$ denotes the numerator of the loop argument which in general depends on the loop momentum. Recursively, all loops can be opened in this way. In each recursion step a loop of $n$ propagators gives $2^{n}-1$ cut diagrams. In practical calculations many of the cut diagrams vanish. Note that in each recursion step the loop order is decreased by at least one unit. Recursive application therefore represents any loop diagram in terms of tree diagrams. In recent years there has been some interest in the Feynman-tree theorem; see for instance [3] 6 . Here we will not consider any modification of the initial theorem. In particular we do not want to restrict the statements to any specific loop order and merely keep the theorem in its general form.

In context with the cuts of the Feynman-tree theorem let us also mention the generalized unitarity method; for a review see for instance [7. The idea of the unitarity method is to construct the amplitude as a function which coincides at all possible cuts. The cut diagrams correspond to known diagrams or are at least diagrams which are easier to calculate. In practical calculations the generalized unitarity method turns out to be very powerful. In contrast, the Feynman-tree theorem expresses any diagram systematically in terms of tree diagrams.

Recently it has been argued that the application of the Feynman-tree theorem followed by BCFW recursion relations [8, 9] gives a systematic way to compute on-shell scattering amplitudes [10, 11]. The BCFW recursion relations split tree diagrams with external on-shell particles into on-shell amplitudes, Opening the loops recursively with the help of

*E-mail: maniatis8@gmail.com 


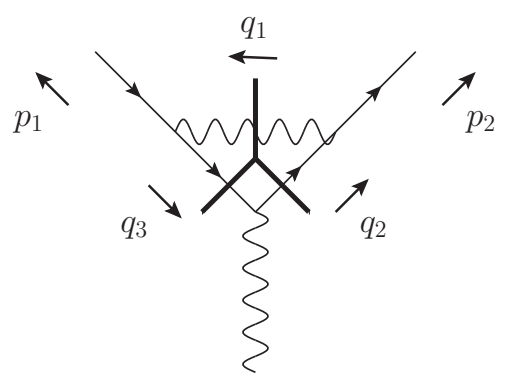

FIG. 1: The cuts of the three propagators of the electron-photon vertex correction. In the Feynman-tree theorem all 7 possible cut combinations have to be applied. The cut propagators correspond to pairs of unobserved particles. Following BCFW these diagrams are given in terms of on-shell amplitudes.

the Feynman-tree theorem followed by BCFW recrusion relations, on-shell and gauge-invariant scattering amplitudes arise in a natural way. Note that neither the Feynman-tree theorem nor the BCFW recursion relation are restricted to four dimensions. Therefore, dimensional regularization can be applied to handle the infrared and ultraviolet singularities. We emphasize that both methods are also not limited to massless particles. Calculations in four dimensions with massless particles have only the advantage, that the Weyl spinor formalism is directly applicable.

In general, in the analytic continuation of the external momenta of the BCFW recursion relations, there may appear non-vanishing boundary terms. However, it has been shown that in gauge theories and gravity this is not the case [12, 13 .

We consider here the explicit calculation of the vertex correction at next-to-leading order in the electromagnetic coupling $e$ for the electron-photon interaction as depicted in Fig. 1. Since this vertex correction involves three propagators, the application of the Feynman-tree theorem gives $2^{3}-1=7$ diagrams; three single-cut diagrams, three double-cut diagrams, and one triple-cut diagram. These diagrams can be computed by on-shell amplitudes following BCFW; see [1] for details. In this paper we shall explicitly calculate all these amplitudes. We have to integrate over the phase space of the corresponding pairs of unobserved particles. The single-cut diagrams have been calculated in the case of scalars [14. Here we consider all cut diagrams and extend these calculations to tensor integrals, which appear in the calculation. We will demonstrate that the phase-space integrations of cut diagrams can be performed and that the sum of all amplitudes yields the well-known result. We emphasize that the essential building blocks are gauge-invariant on-shell amplitudes.

\section{CALCULATION}

Here we present the detailed calculation of the different cut amplitudes contributing to the electron-photon vertex at next-to-leading order in the electromagnetic coupling $e$.

For simplicity, we perform the calculation without considering the mass of the electron. This simplifies the calculations but does not mean any limitation of the method. The phase-space integrations over the unobserved on-shell particle momenta are in general divergent, due to infrared and ultraviolet singularities. Therefore we will regularize the integrations dimensional in $D=4-2 \epsilon$ dimensions, regularizing both, infrared and ultraviolet singularities.

We begin with some general remarks on the phase-space integrations we are going to perform. Let us consider an explicit cut integral, for instance the amplitude coming from the single cut of the photon propagator with momentum $q_{1}$ (see Fig. 1), or equivalently, the amplitude arising from the integration over an unobserved on-shell photon,

$$
A_{1}\left(p_{1}, s_{1}, p_{2}, s_{2}, \lambda\right)=-e^{3} \mu^{4-D} \int \frac{d^{D} l}{(2 \pi)^{D}} 2 \pi \delta^{(+)}\left(q_{1}^{2}\right) \frac{\bar{u}\left(p_{2}, s_{2}\right) \gamma_{\alpha} \phi_{2} \notin(\lambda) q_{3} \gamma^{\alpha} u\left(p_{1}, s_{1}\right)}{\left(q_{2}^{2}+i \epsilon\right)\left(q_{3}^{2}+i \epsilon\right)} .
$$

The minus sign together with the delta distribution originate from the Feynman-tree theorem. The momentum and the spin of the external electrons are denoted by $p_{1 / 2}$ and $s_{1 / 2}$, respectively, whereas $\lambda$ denotes the polarization of the external photon. The (cut) propagator momenta in terms of the integration momentum $l$ are $q_{1}=l, q_{2}=l+p_{2}$, $q_{3}=l-p_{1}$. Since the numerator is not modified by the Feynman-tree theorem (apart from including delta distributions accompanied by $2 \pi$ factors) we encounter in all amplitudes the same numerator $\gamma_{\alpha} q_{2} \gamma_{\mu} q_{3} \gamma^{\alpha}=q_{2}^{\alpha} q_{3}^{\beta}\left(-2 \gamma_{\beta} \gamma_{\mu} \gamma_{\alpha}+(4-\right.$ D) $\gamma_{\alpha} \gamma_{\mu} \gamma_{\beta}$ ), giving tensor integrals of first and second order.

We use the parametrization of the on-shell loop momentum $q_{i}$ as suggested in [14]. In that work also all scalar 
single cut integrals were presented. The explicit parametrization of the on-shell loop momentum reads [14]

$$
q_{i}=\frac{\sqrt{s_{12}}}{2} \xi_{i}\left(1,2 \sqrt{v_{i}\left(1-v_{i}\right)} \boldsymbol{e}_{i, \mathrm{~T}}, 1-2 v_{i}\right)^{\mathrm{T}}
$$

with $\xi_{i} \in\left[0, \infty\left[, v_{i} \in[0,1]\right.\right.$ and $\boldsymbol{e}_{i, \mathrm{~T}}$ a unit vector in transverse direction. In this parametrization we have $q_{i}^{2}=0$ and with $\boldsymbol{p}_{1}$ pointing in positive $z$ direction as well as $\boldsymbol{p}_{2}=-\boldsymbol{p}_{1}$,

$$
2 q_{i} p_{1}=s_{12} \xi_{i} v_{i}, \quad 2 q_{i} p_{2}=s_{12} \xi_{i}\left(1-v_{i}\right) \quad \text { with } \quad s_{12}=\left(p_{1}+p_{2}\right)^{2} .
$$

Shifting the phase-space momentum $l$ to one of the cut momenta we have

$$
d^{D} l=d^{D} q_{i}=d q_{i, 0} d^{D-1} \boldsymbol{q}_{i}=d q_{i, 0} d\left|\boldsymbol{q}_{i}\right|\left|\boldsymbol{q}_{i}\right|^{D-2} d \Omega_{D-1}
$$

with $\Omega_{D}$ the solid angle in $D$ dimensions. With $\left|\boldsymbol{q}_{i}\right|=q_{i, 0}=\frac{\sqrt{s_{12}}}{2} \xi_{i}$ as well as $v_{i}=1 / 2\left(1-\cos \left(\theta_{1}\right)\right)$ we get

$$
d \Omega_{D-1}=\Omega_{D-2} 2^{D-3}\left(v_{i}\left(1-v_{i}\right)\right)^{(D-4) / 2} d v_{i}, \quad \Omega_{D-2}=\frac{2 \cdot \pi^{(D-2) / 2}}{\Gamma((D-2) / 2)} .
$$

We have seen that we have to deal with tensor integrals, arising from the numerator of the integrand in (2.1). Analogously to the tensor reduction of standard integrals [15] we do a covariant decomposition with respect to the available momenta $p_{1}$ and $p_{2}$, explicitly,

$$
I_{j}^{\alpha}=p_{1}^{\alpha} C_{1}+p_{2}^{\alpha} C_{2}, \quad I_{j}^{\alpha \beta}=g^{\alpha \beta} C_{00}+\sum_{i, k=1}^{2} p_{i}^{\alpha} p_{k}^{\beta} C_{i k}
$$

We contract these covariant decompositions with $p_{1, \alpha}$ and $p_{2, \alpha}$ in the case of the tensors of order one, and with $g_{\alpha \beta}$, $p_{1, \alpha} p_{1, \beta}, p_{2, \alpha} p_{2, \beta}, p_{1, \alpha} p_{2, \beta}, p_{2, \alpha} p_{1, \beta}$, respectively, in case of the tensors of second order. Computing these invariants explicitly we determine all the coefficients in $(2.6)$ and in this way the tensor integrals.

With these preparations let us calculate the amplitude $A_{1}, 2.1$, corresponding to a single photon-propagator cut. Let us begin with the calculation of the scalar integral,

$$
I_{1}=-\mu^{4-D} \int \frac{d^{D} l}{(2 \pi)^{D}} 2 \pi \delta^{(+)}\left(q_{1}^{2}\right) \frac{1}{\left(q_{2}^{2}+i \epsilon\right)\left(q_{3}^{2}+i \epsilon\right)} .
$$

We shift the integration variable to the cut momentum $q_{1}$. Integration over the zero component of $q_{1}$ justifies the on-shell parametrization 2.2 . With $q_{2}=q_{1}+p_{2}$ and $q_{3}=q_{1}-p_{1}$, we have $q_{2}^{2}=-2\left(q_{1} p_{2}\right)=-s_{12} \xi_{1}\left(1-v_{1}\right)$, $q_{3}^{2}=-2\left(q_{1} p_{1}\right)=-s_{12} \xi_{1} v_{1}$, and get [14]

$$
I_{1}=\Omega_{D-2} \frac{\mu^{4-D}}{4(2 \pi)^{D-1}} s_{12}^{D / 2-3} \int d \xi_{1} \xi_{1}^{D-5} d v_{1} \frac{\left(v_{1}\left(1-v_{1}\right)\right)^{(D-4) / 2}}{v_{1}\left(1-v_{1}\right)}=0 .
$$

The integration over $\xi_{1}$ in $D$ dimensions is scaleless and therefore has to vanish; for details see for instance 16 . Computing the invariants from $(2.6)$ we see that we always get scaleless integrals and therefore we find $I_{1}^{\alpha}=0$ and $I_{1}^{\alpha \beta}=0$. The photon single-cut amplitude gives no contribution here,

$$
A_{1}\left(p_{1}, s_{1}, p_{2}, s_{2}, \lambda\right)=0
$$

We proceed with the single-cut amplitude integrating over the phase space of the on-shell electron with momentum $q_{2}$,

$$
A_{2}\left(p_{1}, s_{1}, p_{2}, s_{2}, \lambda\right)=-e^{3} \mu^{4-D} \int \frac{d^{D} l}{(2 \pi)^{D}} 2 \pi \delta^{(+)}\left(q_{2}^{2}\right) \frac{\bar{u}\left(p_{2}, s_{2}\right) \gamma_{\alpha} \not q_{2} \notin(\lambda) q_{3} \gamma^{\alpha} u\left(p_{1}, s_{1}\right)}{\left(q_{1}^{2}+i \epsilon\right)\left(q_{3}^{2}+i \epsilon\right)} .
$$

We shift the integration to $q_{2}$ and achieve with $q_{1}^{2}=-s_{12} \xi_{2}\left(1-v_{2}\right)$ and $q_{3}^{2}=s_{12}\left(1-\xi_{2}\right)$ for the scalar integral

$$
\begin{aligned}
I_{2} & =-\mu^{4-D} \int \frac{d^{D} l}{(2 \pi)^{D}} 2 \pi \delta^{(+)}\left(q_{2}^{2}\right) \frac{1}{\left(q_{1}^{2}+i \epsilon\right)\left(q_{3}^{2}+i \epsilon\right)} \\
& =\frac{\mu^{4-D}}{4(2 \pi)^{D-1}} s_{12}^{D / 2-3} \Omega_{D-2} \int d \xi_{2} \frac{\xi_{2}^{D-4}}{1-\xi_{2}+i \epsilon} d v_{2} \frac{\left(v_{2}\left(1-v_{2}\right)\right)^{(D-4) / 2}}{1-v_{2}}
\end{aligned}
$$


The integrals over $\xi_{i}$ and $v_{i}$ can be found in the appendix. We proceed with the tensor integrals 2.6. Decomposing in the numerator of $2.10 q_{2}^{\alpha} q_{3}^{\beta}=q_{2}^{\alpha} q_{2}^{\beta}-q_{2}^{\alpha}\left(p_{1}+p_{2}\right)^{\beta}$ we compute the invariants

$$
\begin{aligned}
& p_{1, \alpha} I_{2}^{\alpha}=\frac{1}{2} \frac{\mu^{4-D}}{4(2 \pi)^{D-1}} s_{12}^{D / 2-2} \Omega_{D-2} \int d \xi_{2} d v_{2} \frac{\xi_{2}^{D-3}}{\left(1-\xi_{2}\right)} \frac{v_{2}}{\left(1-v_{2}\right)}\left(v_{2}\left(1-v_{2}\right)\right)^{(D-4) / 2} \\
& p_{2, \alpha} I_{2}^{\alpha}=\frac{1}{2} \frac{\mu^{4-D}}{4(2 \pi)^{D-1}} s_{12}^{D / 2-2} \Omega_{D-2} \int d \xi_{2} d v_{2} \frac{\xi_{2}^{D-3}}{\left(1-\xi_{2}\right)}\left(v_{2}\left(1-v_{2}\right)\right)^{(D-4) / 2} .
\end{aligned}
$$

With the help of the integrals in the appendix we get the coefficients $C_{1}$ and $C_{2}$ in $(2.6)$, that is, $I_{2}^{\alpha}$.

For the second-order tensor we compute all the corresponding invariants,

$$
\begin{aligned}
& g_{\alpha \beta} I_{2}^{\alpha \beta}=0, \\
& p_{1, \alpha} p_{1, \beta} I_{2}^{\alpha \beta}=\frac{1}{4} \frac{\mu^{4-D}}{4(2 \pi)^{D-1}} s_{12}^{D / 2-1} \Omega_{D-2} \int d \xi_{2} d v_{2} \frac{\xi_{2}^{D-2}}{\left(1-\xi_{2}\right)} \frac{v_{2}^{2}}{\left(1-v_{2}\right)}\left(v_{2}\left(1-v_{2}\right)\right)^{(D-4) / 2}, \\
& p_{2, \alpha} p_{2, \beta} I_{2}^{\alpha \beta}=\frac{1}{4} \frac{\mu^{4-D}}{4(2 \pi)^{D-1}} s_{12}^{D / 2-1} \Omega_{D-2} \int d \xi_{2} d v_{2} \frac{\xi_{2}^{D-2}}{\left(1-\xi_{2}\right)}\left(1-v_{2}\right)\left(v_{2}\left(1-v_{2}\right)\right)^{(D-4) / 2}, \\
& p_{1, \alpha} p_{2, \beta} I_{2}^{\alpha \beta}=p_{2, \alpha} p_{1, \beta} I_{2}^{\alpha \beta}=\frac{1}{4} \frac{\mu^{4-D}}{4(2 \pi)^{D-1}} s_{12}^{D / 2-1} \Omega_{D-2} \int d \xi_{2} d v_{2} \frac{\xi_{2}^{D-2}}{\left(1-\xi_{2}\right)} v_{2}\left(v_{2}\left(1-v_{2}\right)\right)^{(D-4) / 2} .
\end{aligned}
$$

With the explicit $\xi_{i}$ and $v_{i}$ integrations in the appendix we construct the tensor integral $I_{2}^{\alpha \beta}$. In this way we get the amplitude,

$$
A_{2}\left(p_{1}, s_{1}, p_{2}, s_{2}, \lambda\right)=e^{3} \bar{u}\left(p_{1}, s_{1}\right) \notin(\lambda) u\left(p_{2}, s_{2}\right) \frac{2 \pi \mu^{2 \epsilon}}{4(2 \pi)^{3-2 \epsilon}} s_{12}^{-\epsilon} \Omega_{D-2} \frac{\Gamma(2-\epsilon) \Gamma(-\epsilon)}{\Gamma(3-2 \epsilon)}\left(\frac{1}{\tan (2 \pi \epsilon)}-i\right)\left(2-\epsilon+2 \epsilon^{2}\right),
$$

with the solid angle $\Omega_{D-2}$ given in 2.5 .

The amplitude $A_{3}$ reads

$$
A_{3}\left(p_{1}, s_{1}, p_{2}, s_{2}, \lambda\right)=-e^{3} \mu^{4-D} \int \frac{d^{D} l}{(2 \pi)^{D}} 2 \pi \delta^{(+)}\left(q_{3}^{2}\right) \frac{\bar{u}\left(p_{2}, s_{2}\right) \gamma_{\alpha} \not q_{2} \notin q_{3} \gamma^{\alpha} u\left(p_{1}, s_{1}\right)}{\left(q_{1}^{2}+i \epsilon\right)\left(q_{2}^{2}+i \epsilon\right)}
$$

and we compute the scalar integral (see also [14])

$$
\begin{aligned}
I_{3} & =-\mu^{4-D} \int \frac{d^{D} l}{(2 \pi)^{D}} 2 \pi \delta^{(+)}\left(q_{3}^{2}\right) \frac{1}{\left(q_{1}^{2}+i \epsilon\right)\left(q_{2}^{2}+i \epsilon\right)} \\
& =-\frac{\mu^{4-D}}{4(2 \pi)^{D-1}} s_{12}^{D / 2-3} \Omega_{D-2} \int d \xi_{3} d v_{3} \frac{\xi_{3}^{D-4}}{1+\xi_{3}} \frac{\left(v_{3}\left(1-v_{3}\right)\right)^{(D-4) / 2}}{v_{3}}
\end{aligned}
$$

where the integration is shifted to $q_{3}$, with $q_{1}=q_{3}+p_{1}$ and $q_{2}=q_{3}+p_{1}+p_{2}$, that is, $q_{1}^{2}=2 q_{3} p_{1}=s_{12} \xi_{3} v_{3}$ and $q_{2}^{2}=s_{12}\left(1+\xi_{3}\right)$. The tensor in the numerator 2.15 becomes $q_{2}^{\alpha} q_{3}^{\beta}=q_{3}^{\alpha} q_{3}^{\beta}+\left(p_{1}+p_{2}\right)^{\alpha} q_{3}^{\beta}$. We compute the invariants

$$
\begin{aligned}
& p_{1, \alpha} I_{3}^{\alpha}=-\frac{1}{2} \frac{\mu^{4-D}}{4(2 \pi)^{D-1}} s_{12}^{D / 2-2} \Omega_{D-2} \int d \xi_{3} d v_{3} \frac{\xi_{3}^{D-3}}{\left(1+\xi_{3}\right)}\left(v_{3}\left(1-v_{3}\right)\right)^{(D-4) / 2}, \\
& p_{2, \alpha} I_{3}^{\alpha}=-\frac{1}{2} \frac{\mu^{4-D}}{4(2 \pi)^{D-1}} s_{12}^{D / 2-2} \Omega_{D-2} \int d \xi_{3} d v_{3} \frac{\xi_{3}^{D-3}}{\left(1+\xi_{3}\right)} \frac{1-v_{3}}{v_{3}}\left(v_{3}\left(1-v_{3}\right)\right)^{(D-4) / 2} .
\end{aligned}
$$

With the help of the integrals in the appendix we get the coefficients in $(2.6)$, hence, $I_{3}^{\alpha}$.

The invariants for the second-order tensor read

$$
\begin{aligned}
& g_{\alpha \beta} I_{3}^{\alpha \beta}=0, \\
& p_{1, \alpha} p_{1, \beta} I_{3}^{\alpha \beta}=-\frac{1}{4} \frac{\mu^{4-D}}{4(2 \pi)^{D-1}} s_{12}^{D / 2-1} \Omega_{D-2} \int d \xi_{3} d v_{3} \frac{\xi_{3}^{D-2}}{\left(1+\xi_{3}\right)} v_{3}\left(v_{3}\left(1-v_{3}\right)\right)^{(D-4) / 2}, \\
& p_{2, \alpha} p_{2, \beta} I_{3}^{\alpha \beta}=-\frac{1}{4} \frac{\mu^{4-D}}{4(2 \pi)^{D-1}} s_{12}^{D / 2-1} \Omega_{D-2} \int d \xi_{3} d v_{3} \frac{\xi_{3}^{D-2}}{\left(1+\xi_{3}\right)} \frac{\left(1-v_{3}\right)^{2}}{v_{3}}\left(v_{3}\left(1-v_{3}\right)\right)^{(D-4) / 2}, \\
& p_{1, \alpha} p_{2, \beta} I_{3}^{\alpha \beta}=-\frac{1}{4} \frac{\mu^{4-D}}{4(2 \pi)^{D-1}} s_{12}^{D / 2-1} \Omega_{D-2} \int d \xi_{3} d v_{3} \frac{\xi_{3}^{D-2}}{\left(1+\xi_{3}\right)}\left(1-v_{3}\right)\left(v_{3}\left(1-v_{3}\right)\right)^{(D-4) / 2} .
\end{aligned}
$$


We construct the tensor integral $I_{3}^{\alpha \beta}$ from these invariants and get the amplitude,

$$
A_{3}\left(p_{1}, s_{1}, p_{2}, s_{2}, \lambda\right)=e^{3} \bar{u}\left(p_{1}, s_{1}\right) \notin(\lambda) u\left(p_{2}, s_{2}\right) \cdot \frac{2^{2 \epsilon} \pi^{3 / 2} \mu^{2 \epsilon}}{8(2 \pi)^{3-2 \epsilon}} s_{12}^{-\epsilon} \Omega_{D-2} \frac{\Gamma(-\epsilon)}{\Gamma(3 / 2-\epsilon)} \frac{1}{\sin (2 \pi \epsilon)}\left(2-\epsilon+2 \epsilon^{2}\right) .
$$

We proceed with the double-cut amplitude

$$
A_{23}\left(p_{1}, s_{1}, p_{2}, s_{2}, \lambda\right)=e^{3} \mu^{4-D} \int \frac{d^{D} l}{(2 \pi)^{D}} 2 \pi \delta^{(+)}\left(q_{2}^{2}\right) 2 \pi \delta^{(+)}\left(q_{3}^{2}\right) \frac{\bar{u}\left(p_{2}, s_{2}\right) \gamma_{\alpha} \phi_{2} \notin \phi_{3} \gamma^{\alpha} u\left(p_{1}, s_{1}\right)}{q_{1}^{2}+i \epsilon} .
$$

The corresponding scalar integral is, shifting the integration to $q_{3}$,

$$
\begin{aligned}
I_{23} & =\mu^{4-D} \int \frac{d^{D} l}{(2 \pi)^{D}} 2 \pi \delta^{(+)}\left(q_{2}^{2}\right) 2 \pi \delta^{(+)}\left(q_{3}^{2}\right) \frac{1}{q_{1}^{2}+i \epsilon} \\
& =\frac{\mu^{4-D}}{(2 \pi)^{D-2}} \frac{s_{12}^{D / 2-3}}{2^{D-1}} \int d \xi_{3} d \Omega_{D-1} \delta^{(+)}\left(1+\xi_{3}\right) \frac{\xi_{3}^{D-4}}{v_{3}} \\
& =0 .
\end{aligned}
$$

The last step follows from the integration over $\xi_{i} \in[0, \infty]$ and the delta distribution. This result holds also for the corresponding tensor integrals and we find

$$
A_{23}\left(p_{1}, s_{1}, p_{2}, s_{2}, \lambda\right)=0 .
$$

All the other double-cut amplitudes and the triple-cut amplitude vanish, too. In particular, the two remaining double-cut amplitudes turn out to be scaleless and for the triple-cut amplitude, the corresponding scalar integral

$$
I_{123}=\mu^{4-D} \int \frac{d^{D} l}{(2 \pi)^{D}} 2 \pi \delta^{(+)}\left(q_{1}^{2}\right) 2 \pi \delta^{(+)}\left(q_{2}^{2}\right) 2 \pi \delta^{(+)}\left(q_{3}^{2}\right)=0
$$

vanishes since the delta distributions can not be simultaneously fulfilled, like in 2.21). This holds also for the corresponding tensor integrals.

The complete amplitude for the electron-photon vertex correction is therefore the sum of $(2.14)$ and $(2.19)$, expanding around $\epsilon=0$,

$$
\begin{aligned}
A\left(p_{1}, s_{1}, p_{2}, s_{2}, \lambda\right)=\frac{e^{3}}{4} \bar{u}\left(p_{1}, s_{1}\right) \notin(\lambda) u\left(p_{2}, s_{2}\right) \frac{\Omega_{D-2}}{(2 \pi)^{3-2 \epsilon}} . \\
\left\{-\frac{2}{\epsilon^{2}}+\frac{1}{\epsilon}\left(2 \ln \left(\frac{-s_{12}}{\mu^{2}}\right)-3\right)-\ln ^{2}\left(\frac{-s_{12}}{\mu^{2}}\right)+3 \ln \left(\frac{-s_{12}}{\mu^{2}}\right)-8\right\}+\mathcal{O}(\epsilon),
\end{aligned}
$$

which is the well-known result we get in conventional computation from a virtual Feynman diagram loop.

\section{CONCLUSIONS}

The Feynman-tree theorem on the one hand opens recursively all loops of a diagram yielding tree diagrams and on the other hand the BCFW recursion relations transform the tree diagrams into on-shell amplitudes. In this way Feynman diagrams can be decomposed into gauge-invariant on-shell amplitudes. We have considered an explicit example, the electron-photon vertex correction in QED. We encounter phase-space integrations over momenta of unobserved particles arising form single, double, and triple cuts. The tensor integrals have been calculated explicitly. Even though the number of amplitudes originating from the Feynman-tree theorem is in general rather large, we have seen that often multi-cut amplitudes vanish due to the phase-space kinematics. We recovered the well-known result for the electron-photon vertex correction from the calculation of the amplitudes. This demonstrates the consistency of the method to combine the Feynman-tree theorem with BCFW recursion relations, in a physical amplitude. We leave it open for future work to do the calculation with massive particles. Moreover, it would be interesting to show the explicit calculation at higher perturbative order, corresponding in terms of usual Feynman diagrams to higher loop order.

We expect that the combination of the Feynman-tree theorem with the BCFW recursion relations gives some new insights into scattering amplitudes. In particular, it is quite striking that Feynman diagrams in a gauge theory decay into on-shell and gauge-invariant building blocks, which can be calculated separately. 


\section{Acknowledgement}

We would like to thank Simon Caron-Huot and Otto Nachtmann for many valuable comments and suggestions. This work is supported partly by the Chilean research project FONDECYT, with project number 1140568, as well as by the the UBB project GI-152609/VC Física de Altas Energias.

\section{Appendix A: Integrals}

Here we give all the elementary integrals needed in the calculations:

$$
\begin{aligned}
& \int_{0}^{\infty} d \xi \frac{\xi^{2-2 \epsilon}}{1-\xi+i \epsilon}=\int_{0}^{\infty} d \xi \frac{\xi^{1-2 \epsilon}}{1-\xi+i \epsilon}=\int_{0}^{\infty} d \xi \frac{\xi^{-2 \epsilon}}{1-\xi+i \epsilon}=-\int_{-\infty}^{0} d \xi \frac{\xi^{-2 \epsilon}}{1-\xi}=-(-1)^{-2 \epsilon} \frac{\pi}{\sin (2 \pi \epsilon)} \\
& \int_{0}^{\infty} d \xi \frac{\xi^{2-2 \epsilon}}{1+\xi}=-\int_{0}^{\infty} d \xi \frac{\xi^{1-2 \epsilon}}{1+\xi}=\int_{0}^{\infty} d \xi \frac{\xi^{-2 \epsilon}}{1+\xi}=\frac{\pi}{\sin (2 \pi \epsilon)} \\
& \int_{0}^{1} d v(v(1-v))^{-\epsilon}=\frac{\Gamma^{2}(1-\epsilon)}{\Gamma(2-2 \epsilon)} \\
& \int_{0}^{1} d v(v(1-v))^{-\epsilon} \frac{v}{1-v}=\int_{0}^{1} d v(v(1-v))^{-\epsilon} \frac{1-v}{v}=\frac{\Gamma(2-\epsilon) \Gamma(-\epsilon)}{\Gamma(2-2 \epsilon)} \\
& \int_{0}^{1} d v \frac{(v(1-v))^{-\epsilon}}{1-v}=\int_{0}^{1} d v \frac{(v(1-v))^{-\epsilon}}{v}=\frac{\Gamma(1-\epsilon) \Gamma(-\epsilon)}{\Gamma(1-2 \epsilon)} \\
& \int_{0}^{1} d v(v(1-v))^{-\epsilon} \frac{v^{2}}{1-v}=\frac{\Gamma(3-\epsilon) \Gamma(-\epsilon)}{\Gamma(3-2 \epsilon)} \\
& \int_{0}^{1} d v(v(1-v))^{-\epsilon} \frac{(1-v)^{2}}{v}=\frac{\sqrt{\pi}}{4} 4^{\epsilon}(2-\epsilon) \frac{\Gamma(-\epsilon)}{\Gamma(3 / 2-\epsilon)} \\
& \int_{0}^{1} d v(v(1-v))^{-\epsilon} v=\frac{\Gamma(1-\epsilon) \Gamma(2-\epsilon)}{\Gamma(3-2 \epsilon)} \\
& \int_{0}^{1} d v(v(1-v))^{-\epsilon}(1-v)=\frac{\sqrt{\pi}}{4} 4^{\epsilon} \frac{\Gamma(1-\epsilon)}{\Gamma(3 / 2-\epsilon)}
\end{aligned}
$$

[1] R. P. Feynman, "Quantum theory of gravitation," Acta Phys. Polon. 24, 697 (1963).

[2] R. P. Feynman, "Closed Loop And Tree Diagrams," in Selected papers of Richard Feynman, ed. L. M. Brown (World Scientific, Singapore, 2000).

[3] A. Brandhuber, B. Spence and G. Travaglini, "From trees to loops and back," JHEP 0601, 142 (2006) arXiv:hepth/0510253.

[4] S. Catani, T. Gleisberg, F. Krauss, G. Rodrigo and J. C. Winter, "From loops to trees by-passing Feynman's theorem," JHEP 0809, 065 (2008) arXiv:0804.3170 [hep-ph]].

[5] S. Caron-Huot, "Loops and trees," JHEP 1105, 080 (2011) arXiv:1007.3224 [hep-ph]].

[6] I. Bierenbaum, S. Catani, P. Draggiotis and G. Rodrigo, "A Tree-Loop Duality Relation at Two Loops and Beyond," JHEP 1010, 073 (2010) arXiv:1007.0194 [hep-ph]].

[7] Z. Bern and Y. t. Huang, J. Phys. A 44, 454003 (2011) arXiv:1103.1869 [hep-th]].

[8] R. Britto, F. Cachazo and B. Feng, "New recursion relations for tree amplitudes of gluons," Nucl. Phys. B 715, 499 (2005) arXiv:hep-th/0412308.

[9] R. Britto, F. Cachazo, B. Feng and E. Witten, "Direct proof of tree-level recursion relation in Yang-Mills theory," Phys. Rev. Lett. 94, 181602 (2005) arXiv:hep-th/0501052.

[10] M. Maniatis, "Scattering amplitudes abandoning virtual particles," arXiv:1511.03574 [hep-th].

[11] M. Maniatis and C. M. Reyes, "Scattering amplitudes from a deconstruction of Feynman diagrams," arXiv:1605.04268 [hep-th].

[12] N. Arkani-Hamed and J. Kaplan, "On Tree Amplitudes in Gauge Theory and Gravity," JHEP 0804, 076 (2008) arXiv:0801.2385 [hep-th]].

[13] B. Feng, J. Wang, Y. Wang and Z. Zhang, "BCFW Recursion Relation with Nonzero Boundary Contribution," JHEP 1001, 019 (2010) arXiv:0911.0301 [hep-th]].

[14] R. J. Hernandez-Pinto, G. F. R. Sborlini and G. Rodrigo, "Towards gauge theories in four dimensions," JHEP 1602, 044 (2016) arXiv:1506.04617 [hep-ph]]. 
[15] G. Passarino and M. J. G. Veltman, "One Loop Corrections for e+ e- Annihilation Into mu+ mu- in the Weinberg Model," Nucl. Phys. B 160, 151 (1979).

[16] A. Pak and A. Smirnov, "Geometric approach to asymptotic expansion of Feynman integrals," Eur. Phys. J. C 71, 1626 (2011) arXiv:1011.4863 [hep-ph]]. 